\title{
The effect of cationic surfactant and some organic/inorganic additives on the morphology of mesostructured silica templated by pluronics
}

\author{
Altug S. Poyraz, Cemal Albayrak, Ömer Dag* \\ Laboratory for Advanced Functional Materials, Department of Chemistry and Institute of Materials Science and Nanotechnology-UNAM, Bilkent University, 06800 Ankara, Turkey
}

\section{A R T I C L E I N F O}

\section{Article history:}

Received 7 January 2008

Received in revised form 25 February 2008

Accepted 26 February 2008

Available online 4 March 2008

\section{Keywords:}

Mesoporous silica

Spheres

Wormlike particles

Cationic-pluronic interactions

Morphology

\begin{abstract}
A B S T R A C T
Tri-block copolymers (poly(ethylene oxide)-poly(propylene oxide)-poly(ethylene oxide), represented as $\left.\mathrm{EO}_{x} \mathrm{PO}_{y} \mathrm{EO}_{x}\right)$, pluronics $\left(\mathrm{F} 127=\mathrm{EO}_{106} \mathrm{PO}_{70} \mathrm{EO}_{106}, \quad \mathrm{P} 65=\mathrm{EO}_{20} \mathrm{PO}_{30} \mathrm{EO}_{20}, \quad \mathrm{P} 85=\mathrm{EO}_{27} \mathrm{PO}_{39} \mathrm{EO}_{27}, \quad \mathrm{P} 103=\right.$ $\mathrm{EO}_{17} \mathrm{PO}_{55} \mathrm{EO}_{17}$, and $\mathrm{P} 123=\mathrm{EO}_{20} \mathrm{PO}_{70} \mathrm{EO}_{20}$ ) and cationic surfactants (cethyltrimethylammonium bromide (CTAB)), two surfactant systems, form complex micelles that self-assemble into mesostructured particles with distinct morphology depending on the pluronic type, the concentration of the cationic surfactant and the organic-inorganic ingredients in a siliceous reaction media under acidic conditions. The CTABP65 and CTAB-P85 systems form spheres, CTAB-P103 and CTAB-P123 systems form wormlike particles, and CTAB-F127 system form single crystals of mesostructured silica particles under very similar conditions. However addition of various salts (such as $\mathrm{KCl}$ and $\mathrm{NaNO}_{3}$ ) into a CTAB-P103 or CTAB-P123 solution system and cyclohexane and $\mathrm{KCl}$ into a CTAB-P85 solution system produces the mesostructured silica spheres and wormlike particles, respectively. By controlling the hydrophilic-hydrophobic character of the pluronics, core-corona interface, by means of additives, such as small organic molecules or salts, one could obtain the desired morphology that is dictated by the shape of the micelles of the pluronic-cationic surfactant complex. The effects of the additives and the formation mechanism of those morphologies have been discussed using spectroscopy (FT-IR and Raman), diffraction (XRD) and microscopy (POM and SEM) data.
\end{abstract}

(c) 2008 Elsevier Inc. All rights reserved.

\section{Introduction}

Since the discovery of mesoporous silica [1,2], there has been a tremendous effort devoted to functionalizing the mesoporous materials either by surface modification using various active chemicals or by controlling their morphology. Many research groups have synthesized spherical mesoporous silica [3-10] in all sizes for various purposes, from drug delivery systems to photonic crystals. Yano et al. [3] have established a method of making nanospheres with uniform size distribution using cationic surfactant in a water/methanol solution in basic media. Mesa et al. [8] have used pluronics, P123 and F127, to produce spherical beads using a cationic surfactant as a co-surfactant. However, in the cationic surfactant/pluronic systems, it is difficult to obtain a uniform size distribution [8-10] and yet the role of the cationic surfactant is not clarified.

Ozin et al. [11,12] investigated a surfactant-silica chemistry that yields various shapes including gyroids, ropes, toroids, discoids, shells, knots etc. Many groups have latter established methods to synthesize one shape in one pot [3-10,13-16]. Tang et al.

\footnotetext{
* Corresponding author. Tel.: +90 312290 3918; fax: +90 3122664068 .

E-mail address: dag@fen.bilkent.edu.tr (Ö. Dag).
}

[13] have systematically investigated the topological transformation of mesostructured silica from gyroids to spheres using three surfactant systems, cationic (CTAB), anionic (Sodium Dodecyl Sulphate, SDS) and non-ionic (P123) and a co-surfactant (ethylacetate, EA). Three surfactant systems, CTAB-SDS-P123 and CTAB-SDSP123-EA have been used to make spheres, ropes, and helical mesostructured silica materials [13,14]. Yang et al. [15] investigated a similar transformation of a rod-like to a vesicle-like mesoporous silica by increasing the hydrophobic content of the media in a triisopropylbenzene-P123 system. Stucky et al. [17] investigated the formation of single crystals and ropes from P108 and P123 systems, respectively, using various salts.

Synthesizing a single shape in one pot is important for many applications, such as catalysis, adsorption, separation, sensors and as a host for CVD for various purposes (optic, magnetic, photonic etc.). There are many efforts towards controlling the morphology [3-10,16,17]. Controlling the salt type and the salt concentration influences both the morphology [16] and structure of the mesophases [18-20]. The presence of salt ions, especially the anions, influences the hydrophilicity of the pluronics both in liquid and liquid crystalline media [18-20]. We have investigated how the nitrate and perchlorate ions change the hyrophilic-hydrophobic balance of the pluronics during the synthesis of mesostructured silica films 
and monoliths [18]. Other than a few studies, [13-15] the current literature mainly focus on finding reaction conditions to produce one shape or to obtain uniform particle size distribution in one pot synthesis.

In this contribution, we have synthesized mesostructured silica spheres, wormlike particles and single crystals using P65, P85, P103, P123 and F127 with CTAB as a co-surfactant in an acidic media. Most of these samples have been synthesized for the first time, and investigated the role of CTAB molecules and some additives (such as salts and cyclohexane) using PXRD, SEM, EDS, FTIR and Raman spectroscopy techniques.

\section{Experimental section}

\subsection{Synthesis}

The procedure established by Mesa et al. [8] or its modified version have been used for the synthesis of mesoporous silica with different morphologies. By using a similar procedure mesostructured silica spheres, wormlike particles and single crystals were synthesized using P85, P65, P123, P103, and F127 tri-block copolymers with a cationic surfactant (CTAB). In a typical synthesis procedure, $0.123 \mathrm{~g}$ of cetyhltrimethylammonium bromide (CTAB) was dissolved in a $0.14 \mathrm{M} 50 \mathrm{ml} \mathrm{HCl}$ solution by continuous stirring. To this solution, a $0.620 \mathrm{~g}$ of $\mathrm{P} 85\left(\mathrm{EO}_{27} \mathrm{PO}_{33} \mathrm{EO}_{27}\right)$ was added and slowly heated while stirring to obtain a clear solution. The experiments containing additional ingredients were conducted by following the same procedure. In these experiments various salts $\left(\mathrm{KCl}\right.$ or $\left.\mathrm{NaNO}_{3}\right)$ and organic molecules (cyclohexane) were added and completely dissolved before the addition of pluronic surfactant. Then the clear solution was cooled to room temperature (RT) and then a $1.25 \mathrm{~g}$ tetramethylorthosilicate (TMOS) was added by vigorous stirring. The final solution was stirred for another $3 \mathrm{~min}$ at RT and then kept in an oven at $95{ }^{\circ} \mathrm{C}$ for 5 days. The precipitate obtained from the above mixture was filtered, washed with excess water and kept in an oven at $60^{\circ} \mathrm{C}$ to dry. Other compositions, changes in the $\mathrm{CTAB} /$ pluronic mole ratios, the amount of added salts and organic molecules have been described in the results and discussion section and tabulated in Table 1.

Table 1

The type of pluronics, pluronic/acid/TMOS amounts, CTAB/Pluronic mole ratio, additive concentration used in the procedure given in the experimental section and the observed morphologies

\begin{tabular}{|c|c|c|c|}
\hline Pluronics & $\begin{array}{l}\mathrm{CTAB} / \text { Pluronic (mole } \\
\text { ratio) }\end{array}$ & Additives & Morphology \\
\hline P65 & $0.00-3.00$ & & Sphere \\
\hline \multirow[t]{9}{*}{ P85 } & 0.00 & & Disordered \\
\hline & 0.18 & & Wormlike \\
\hline & 0.61 & & Wormlike and sphere \\
\hline & 1.22 & & Sphere \\
\hline & $4.00-6.64$ & & Sphere and films \\
\hline & 10.0 & & Monoliths \\
\hline & 2.50 & $\begin{array}{l}5 \mathrm{ml} \\
\text { Cyclohexane }\end{array}$ & Wormlike \\
\hline & 2.50 & $1 \mathrm{~g} \mathrm{KCl}$ & Wormlike \\
\hline & 2.50 & $1 \mathrm{~g} \mathrm{NaNO}_{3}$ & Sphere \\
\hline P103 & 3.34 & & Wormlike \\
\hline P123 & 2.00 & & Wormlike \\
\hline \multirow[t]{3}{*}{ F127 } & $0.0-1.67$ & & Disordered \\
\hline & 2.50 & & $\begin{array}{l}\text { Rhombododecahedron (major } \\
\text { particles) }\end{array}$ \\
\hline & Above 5.00 & & $\begin{array}{l}\text { Rhombododecahedron (only } \\
\text { product) }\end{array}$ \\
\hline
\end{tabular}

The pluronic (grams)/acid (molarity, ml)/TMOS (gram) amounts are $0.62 / 0.14 \mathrm{M}$ $50 \mathrm{ml} / 1.23$ in all samples.

\subsection{Characterization}

The powder X-Ray diffraction (PXRD) patterns were collected on a Rigaku Miniflex diffractometer using a high power $\mathrm{Cu} \mathrm{Ka}$ source operating at $30 \mathrm{kV} / 15 \mathrm{~mA}$. The XRD patterns of the samples were collected in the $1-10,2 \theta$ range with a scan rate of
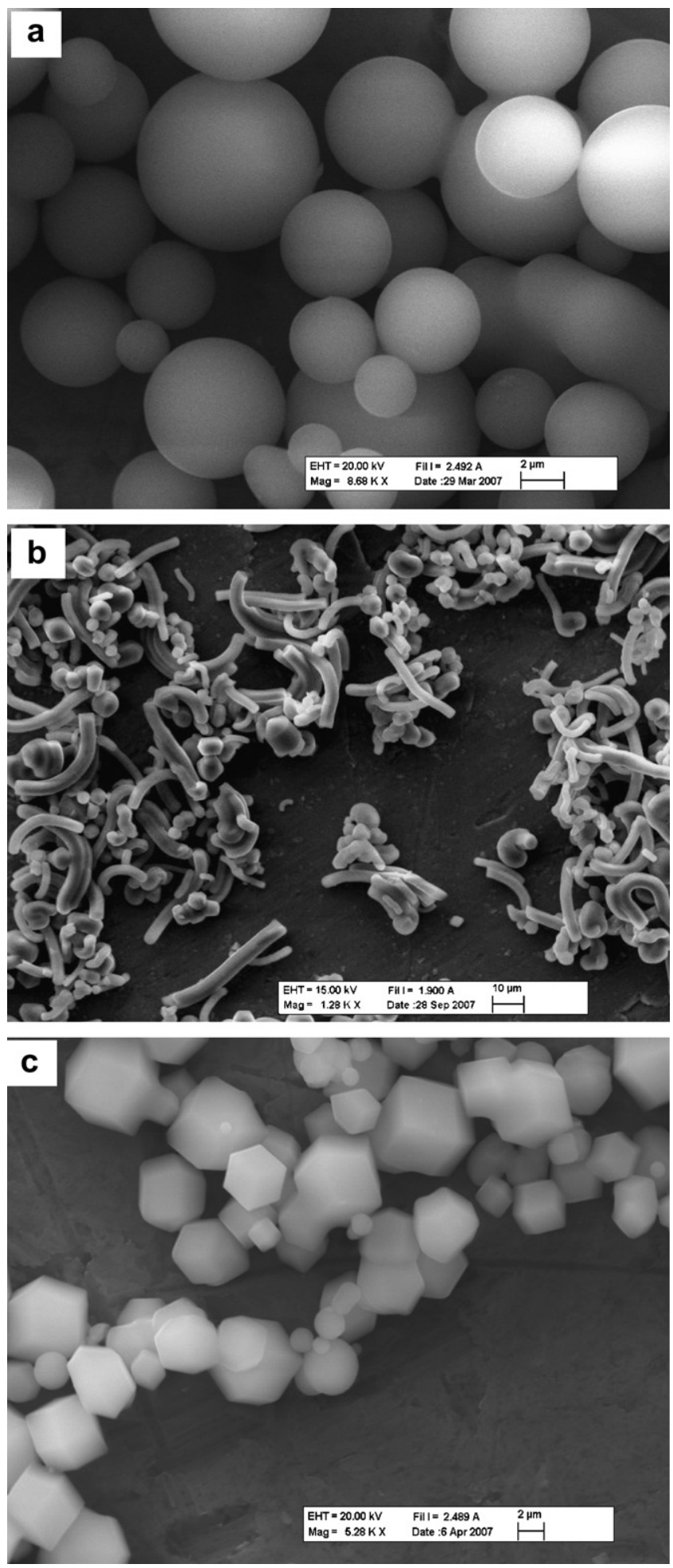

Fig. 1. The SEM images of mesostructured silica particles synthesized using (a) CTAB-P85 (CTAB/P85 mole ratio of 2.5), (b) CTAB-P103 (CTAB/P103 mole ratio of 3.0), and (c) CTAB-F127 (CTAB/F127 mole ratio of 5.0) 
$0.5^{\circ} / \mathrm{min}$. The polarized optical microscopy (POM) images were recorded in transmittance mode on a Meije techno ML 9400 series Polarizing Microscope with transmitted light illumination, using convergent white light between parallel and cross polarizers. The FTIR spectra were recorded using a Bruker Tensor 27 model FTIR spectrometer. A DigiTectTM DLATGS detector was used with a resolution of $4 \mathrm{~cm}^{-1}$ and 256 scans in the $400-$ $4000 \mathrm{~cm}^{-1}$ range for all samples. The micro-Raman spectra were recorded on a LabRam confocal Raman microscope with a $300 \mathrm{~mm}$ focal length. The spectrometer is equipped with a HeNe laser operated at $20 \mathrm{~mW}$, polarized 500:1 with a wavelength of $632.817 \mathrm{~nm}$, and a $1024 \times 256$ element CCD camera. The scanning electron microscopy (SEM) images of all samples were recorded on a ZEISS EVO40. The samples were prepared by dispersing the powders onto aluminum holders using acetone. Energy dispersive X-Ray scattering (EDS) maps were collected using the same SEM using Bruker AXS XFlash detector 4010.

\section{Results and discussion}

We have applied a modified version of the methods established by Mesa et al. [8] in the CTAB-Pluronic systems to synthesize mesostructured silica particles. In this investigation, five different pluronics, P65, P85, P103, P123, and F127 have been used with CTAB as a co-surfactant. The $\mathrm{CTAB} /$ Pluronic mole ratio was varied from 0.0 to 10.0 to optimize the reaction conditions and to elucidate the effect of CTAB in the formation of the mesostructured silica particles. The as-synthesized samples after washing and drying process were characterized by microscopy, spectroscopy and diffraction methods. Fig. 1 display the SEM images of the as-synthesized materials. The images clearly show that the particles are spherical in case of the CTAB-P65 (not shown) and CTAB-P85 systems, wormlike in the case of CTAB-P103 and CTAB-P123 (not shown) systems and single crystalline in the CTAB-F127 system in acidic media under our reaction conditions, Fig. 1 . The wormlike morphologies from
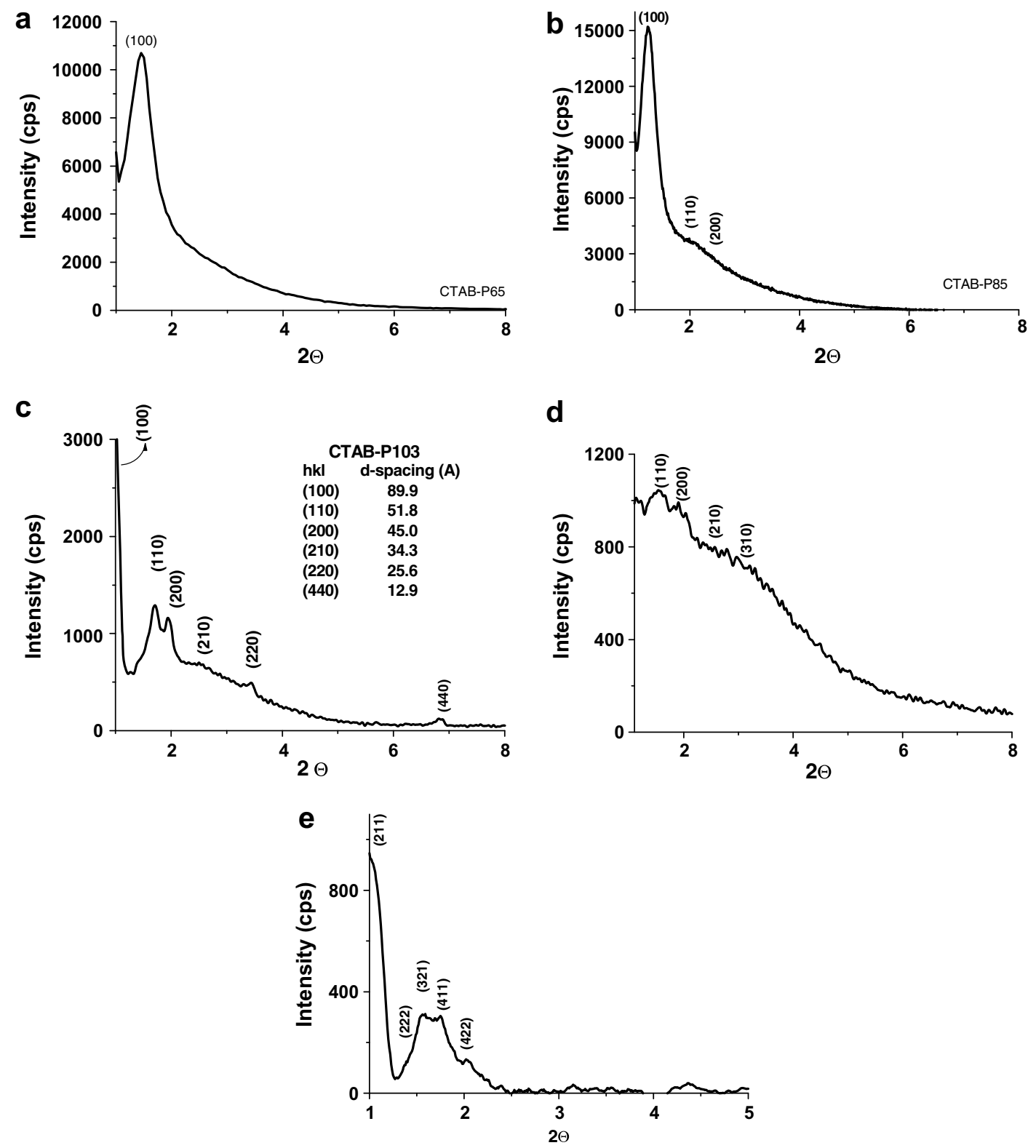

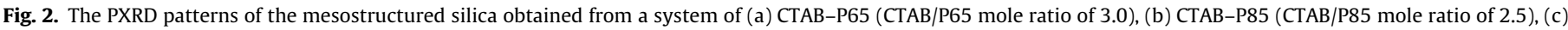
CTAB-P103 (CTAB/P103 mole ratio of 3.3), (d) CTAB-P123 (CTAB/P123 mole ratio of 2.0) and (e) CTAB-F127 (CTAB/F127 mole ratio of 6.0). 
a CTAB-P85 system or spherical morphology from a CTAB-P123 can also be obtained upon the addition of an additive to the reaction media (see text latter). This control was achieved either by adding non-polar ingredients or alkaline metal salts. Each shape can be obtained from a CTAB-Pluronic couple in a broad range of $\mathrm{CTAB} /$ Pluronic mole ratios.

Fig. 2 shows the PXRD patterns of various samples prepared using five different pluronics in the presence of various amounts
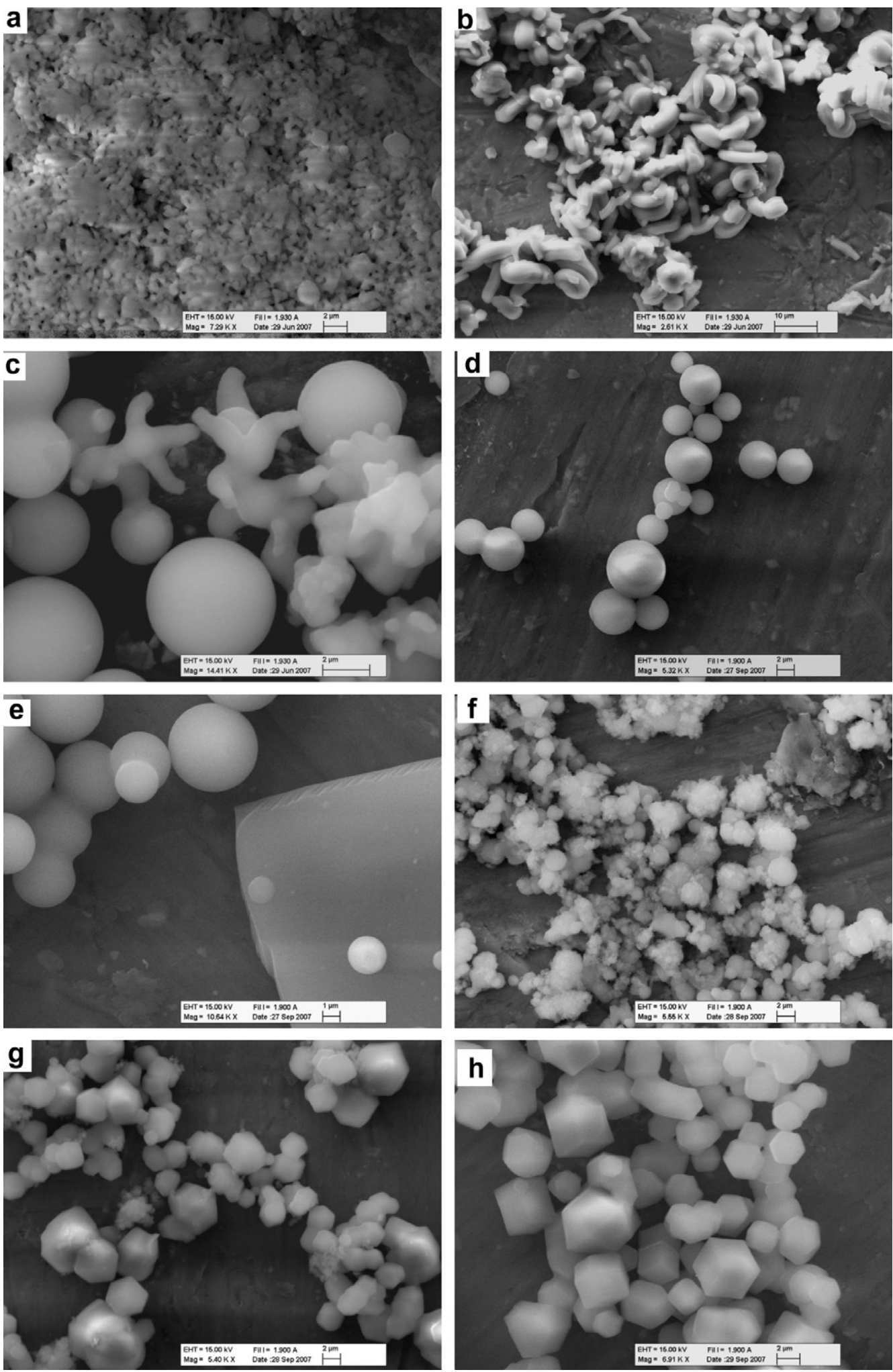

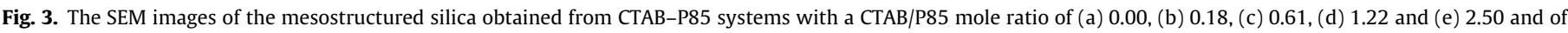
CTAB-F127 with a CTAB/F127 mole ratio of (f) 1.67 , (g) 3.33, and (h) 5.00 
of CTAB. The use of P65, P85, P103, and P123 enables us to tune the unit cell diameters in the spheres in a broad range, from 6 to $10 \mathrm{~nm}$. The CTAB-P65 or CTAB-P85 systems and the CTAB-P103 or CTAB-P123 systems produce $2 \mathrm{D}$ hexagonal mesostructures with a $\mathrm{P} 6 \mathrm{~mm}$ space group with either spherical or wormlike morphologies, respectively. However, only the wormlike structures are birefringent under POM between the crossed polarizers, indicating that the channels are oriented along the rope axis rather than random as in the spheres. The samples obtained from the CTAB-P103 system display up to six diffraction lines, which can be indexed to (100), (110), (200), (210), (220), and (440) planes of 2D hexagonal structure with a P6mm space group, Fig. 2. The mesostructured CTAB-F127 samples have a crystalline morphology with a rhombododecahedron shape. The XRD pattern of the crystalline sample has diffraction lines at $2.04,1.76,1.56,1.44,1.02^{\circ}, 2 \theta$ corresponding to (422), (411), (321), (222), and (211) reflections of Im3m space group of cubic symmetry with a unit cell parameter of $a$ equals to $21.2 \mathrm{~nm}$. Note also that the samples prepared without CTAB do not diffract at small angles, indicating that the mesostructure is disordered or does not form in the absence of CTAB under our reaction conditions.

The effect of CTAB to the CTAB-P85 and CTAB-F127 systems has been investigated by carrying out the following experiments. The cationic surfactant to pluronic mole ratio was varied from 0.0 to 6.6 in the CTAB-P85 and 0.0 to 10.0 in CTAB-F127. In the absence of the cationic surfactant, one gets aggregated small particles with a distinct but not well defined morphology, Fig. 3a. In the presence of enough $\mathrm{CTAB}$, at lower $\mathrm{CTAB}$ concentrations, the particles are only wormlike (CTAB/P85 mole ratio of $0.1-0.6)$, spherical at intermediate concentrations (CTAB/P85 mole ratio of $0.8-4.0)$ and a mixture of films and spheres at high concentrations (CTAB/P85 mole ratio of 4.0-6.6), Fig. 3. The addition of a small amount of CTAB prevents aggregation of the particles indicating that the $\mathrm{CTA}^{+}$ions are also on the surface of the growing particles. The $\mathrm{CTA}^{+}$ions form a complex at intermediate concentrations (CTAB) P85 mole ratio of $0.8-2.5$ ) and the excess $\mathrm{CTA}^{+}$ions go to the air-water interface to produce mesostructured silica films, Fig. 3e. Note that the samples prepared using high CTAB concentrations contain a large amount of film samples, formed in the air water interface. Those film samples diffract at small angles characteristic of a film samples prepared using pure CTAB. If one prepares

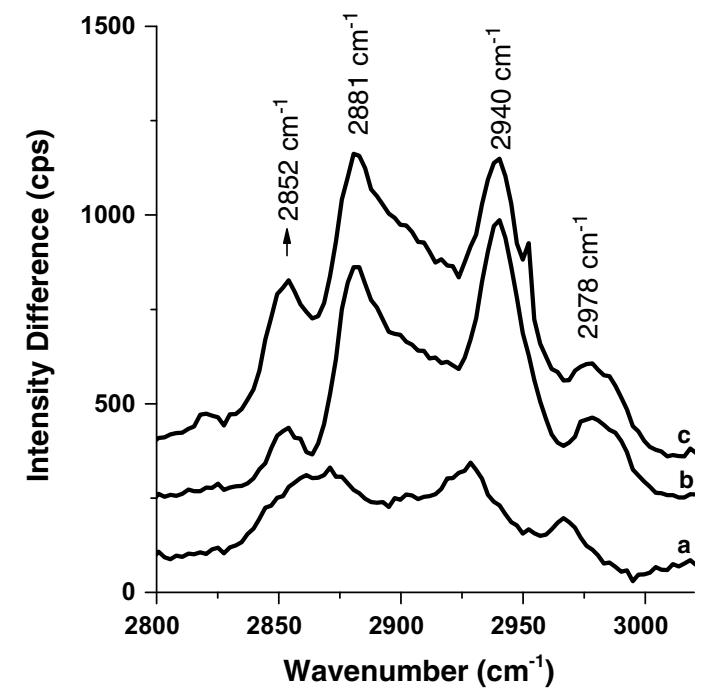

Fig. 4. Micro-Raman difference spectra between mesostructured silica obtained from CTAB-P85 systems and pure P85 $(\mathrm{CTAB} / \mathrm{P} 85=0.0, \mathrm{X})(\mathrm{a})$ the CTAB/P85 mole ratios of $0.46-\mathrm{X}$, (b) CTAB/P85 mole ratios of $1.66-\mathrm{X}$, and (c) CTAB/P85 mole ratios of $3.33-\mathrm{X}$. with even larger quantities of $\mathrm{CTAB}$, one obtains monoliths at the bottom of the reaction vessel. Therefore, we did not further investigate those samples.

Fig. 4 displays the Raman difference spectra of the silica spheres obtained from the CTAB-P85 (CTAB/P85 mole ratio of $0.46,1.66$ and 3.33) system and silica particles obtained from pure P85 system under similar reaction conditions. Notice that the peaks marked on the difference spectra at $2852 \mathrm{~cm}^{-1}$ correspond to the dissolved CTAB (see later) and all the other peaks at 2881, 2940 and $2978 \mathrm{~cm}^{-1}$ correspond to asymmetric $v-\mathrm{CH}_{2}$, symmetric and asymmetric $v-\mathrm{CH}_{3}$ groups of the PO units [21], respectively. The spectral changes are in the PO block of the P85 molecules and the dissolved CTAB signals become visible in the difference spectra, which suggests that the $\mathrm{CTA}^{+}$ions interact with the PO units of the P85 molecules and are involved in the assembly process. Most likely, the interaction of the alkyl tail of the CTAB molecules within the PO core of the CTAB-P85 micelles influences the conformation on the PO blocks and as a result alters the intensity of the $\mathrm{CH}_{2}$ and $\mathrm{CH}_{3}$ groups of the PO units. We have further investigated the CTAB-P85 system in various conditions to discover the interactions between the CTAB and P85 and also to determine the functions of the $\mathrm{CTA}^{+}$ions in the mesostructured silica assembly process.

The spectral changes, shown in Fig. 4 and the observed morphology, shown in Fig. 3a-e, are consistent with each other such that, the difference spectra display almost no change above 3.33 $\mathrm{CTAB} / \mathrm{P} 85$ mole ratios, indicating that the excess CTAB molecules are not part of the mesostructured silica. Moreover, in the CTABF127 systems, one needs more cationic surfactant to observe the effect. The single crystals form at a CTAB/F127 mole ratio as low as 3.33 , but the pure crystals form at higher concentrations, CTAB/F127 mole ratio of 5.0 and above, Fig. 3f-5h. The CTAB$\mathrm{P} 123$ and CTAB-P103 systems usually produce wormlike morphology in a broad range of CTAB concentrations. However at higher $\mathrm{CTAB} /$ Pluronic ratios, the spherical particles can also be obtained from the CTAB-P123 (CTAB/P123 mole ratio of 5.0) and CTABP103 (CTAB/P103 mole ratio of 3.3) systems.

The spherical morphology is usually obtained at relatively lower CATB/Pluronic mole ratios in smaller pluronics, such as $\mathrm{P} 65$ versus P85 and P103 versus P123. The CTAB-P85 system also produce wormlike particles if the CTAB/P85 mole ratio is below 0.5 , however the CTAB-P65 system gives spherical particles at a mole ratio as low as 0.1 . Similarly, the $\mathrm{CTAB} / \mathrm{P} 103$ gives only spherical particles, if the CTAB/P103 mole ratio is higher than 2.5. However, the spherical and wormlike particles with a much smaller aspect ratio coexist in the CTAB-P123 system with a CTAB/P123 mole ratio as high as 3.33. In all CTAB-Pluronic systems, the $\mathrm{CTA}^{+}$ions influence the hydrophilicity of the pluronics by incorporating the alkyl tail of the cationic surfactant into the polypropylene oxide core and carrying the negatively charged counter anions together to the polypropylene oxide-polyethylene oxide region of the pluronics. This makes the PPO-PEO interface more hydrophilic, see Scheme 1. Notice that the PEO/PPO mole ratio is higher, 1.64 and 1.33, in both P65 and P85, respectively, than that of 0.62 and 0.57 in P103 and P123, respectively. Therefore in both P65 and P85 systems, the media is hydrophilic enough, such that there is no need for large amounts of cationic surfactant to produce spherical particles. However, F127 is even more hydrophilic than P65 and P85, still one needs high CTAB concentrations in the CTAB-F127 system, there-

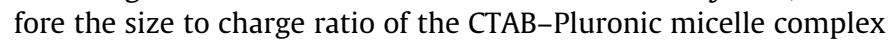
is also as important.

The interaction and formation of a complex between cationic surfactant and pluronics, were further investigated using Raman and FTIR spectroscopy methods. Fig. 5a and b display a series of Raman spectra of a water-CTAB, water-P85, and water-CTAB-P85 systems in various stages of the water evaporation. Notice that 

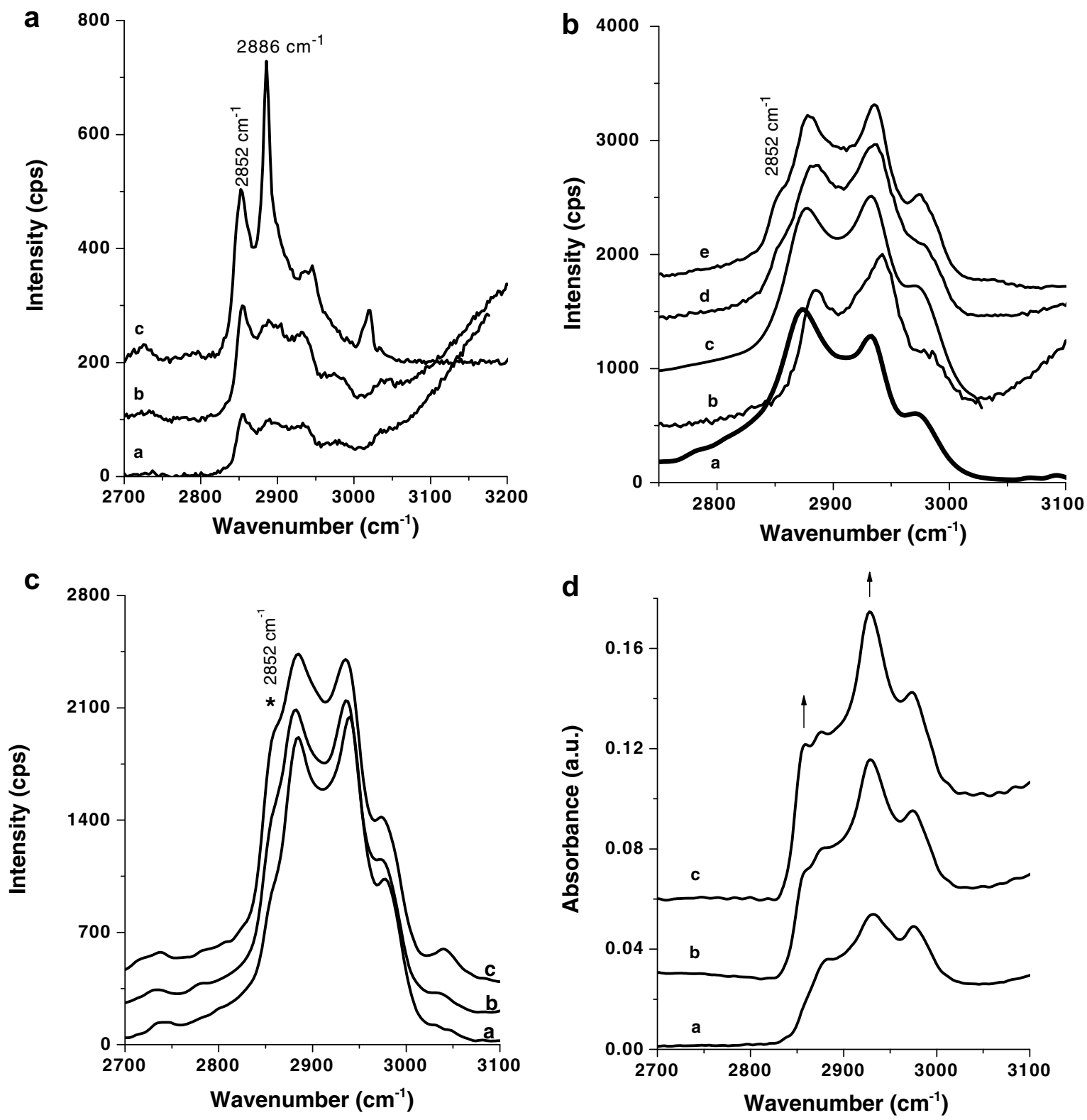

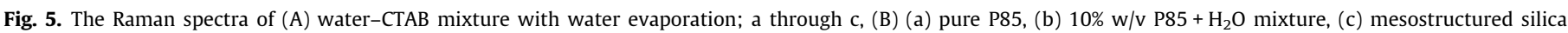

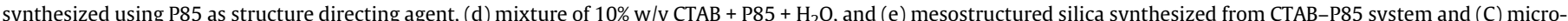
Raman and (D) FTIR spectra of mesostructured (a) CTAB-P65-SiO ${ }_{2}$, (b) $\mathrm{KCl}-\mathrm{CTAB}-\mathrm{P} 65-\mathrm{SiO}_{2}$ and (c) $\mathrm{NaNO}_{3}-\mathrm{CTAB}^{-\mathrm{P} 65-\mathrm{SiO}} 2$.

the spectra of the water-CTAB changes drastically upon evaporation of water, Fig. 5a. The peak at around $2886 \mathrm{~cm}^{-1}$ that originates from the asymmetric stretching mode of $\mathrm{CH}_{2}$ groups of $\mathrm{CTA}^{+}$ion is very sensitive to dissolution. The spectrum of the CTAB-P85 complex (see Scheme 1) in the presence of a small amount of water closely resembles that of the spectrum of the mesoporous silica materials prepared using CTAB and P85, indicating that both surfactants are present in the channels. Note also that the spectrum of the dissolved P85 is quite different from the spectra that contain CTAB and P85 mixtures. The peak at $2852 \mathrm{~cm}^{-1}$ appears only in the presence of $C T A B$ in all spectra (the spectra of CTAB-P85- $\mathrm{H}_{2} \mathrm{O}$, CTAB-P85- $\mathrm{SiO}_{2}$ and CTAB-P85- $\left[\mathrm{Zn}\left(\mathrm{H}_{2} \mathrm{O}\right)_{6}\right]\left(\mathrm{NO}_{3}\right)_{2}$ mixtures) without the intense asymmetric stretching mode of the $\mathrm{CH}_{2}$ units of CTAB crystals at around $2885 \mathrm{~cm}^{-1}$, indicating that the CTAB is completely dissolved in all cases, in a form of a CTAB-P85 complex. The FTIR spectra of the mesoporous silica prepared using P85 in the presence and absence of CTAB are also presented in Fig. 5b for comparison purpose. Notice that these two spectra differ slightly from one another. The difference spectrum of the two spectra shows peak related to the dissolved CTAB. Based on our IR and Raman observations we suggest that the CTAB-P85 complex in the micelle form before interaction with the silica species in the selfassembly process. The complexing of CTAB-Pluronics makes the micelles positively charged, thus enhances the surfactant-silica assembly process.

As discussed above, the CTAB molecules play important roles in the silica assembly process. The formation of a complex between the $\mathrm{CTA}^{+}$ions and pluronic provides positive charges to each micelle, Scheme 1 . Therefore, the unit cell parameters of the particles are determined by the size of the pluronic micelles, Fig. 2. The complex formation has been investigated using FTIR and Raman spectroscopy techniques in this work using as-synthesized 


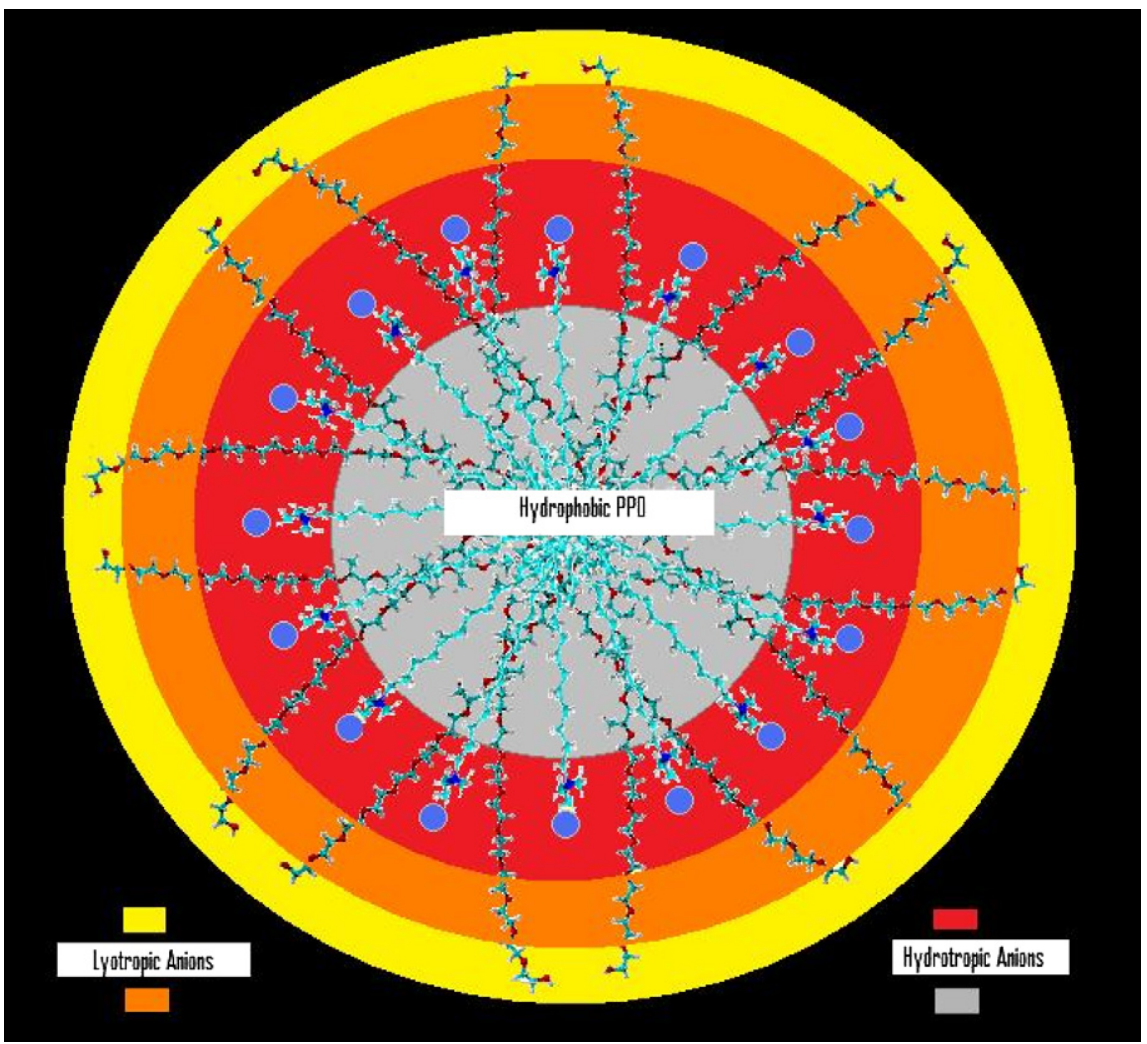

Scheme 1.

mesostructured silica particles, water-CTAB, water-CTAB-P85, and CTAB-P85-[Zn( $\left.\left(\mathrm{H}_{2} \mathrm{O}\right)_{6}\right]\left(\mathrm{NO}_{3}\right)$. Note also that the CTAB-P85$\left[\mathrm{Zn}\left(\mathrm{H}_{2} \mathrm{O}\right)_{6}\right]\left(\mathrm{NO}_{3}\right)$ mixture form liquid crystalline mesophases that closely resembles the structure of mesostructured silica, CTAB$\mathrm{P} 85-\mathrm{SiO}_{2}$. Note that the interaction of surfactants (non-ionic, cationic and anionic) with pluronics has been widely investigated in aqueous solutions and in liquid crystalline phases using light scattering, calorimetry, small-angle X-ray scattering and NMR techniques [22-26]. It is a common conclusion that the cationic surfactant molecules interact with pluronics to form charged complex micelles in an aqueous media [22-24], which is consistent with our proposal.

It is known that the presence of anions also influences the conformation, as a result the hydrophilicity, of the ethylene oxide groups of the pluronics [27-29]. The anions of metal salts influence the solubility, conformation of the surfactants in both liquid and liquid crystalline media [27-29]. It has also been shown that the anions of the salts have a great effect on the morphology of the mesostructured silica in dilute and concentrated media $[14,16,30]$. Some anions, on the left hand side of the Hofmeister's series [31] $\left(\mathrm{SO}_{4}^{2-}>\mathrm{HPO}_{4}^{2-}>\mathrm{CrO}_{4}^{-}>\mathrm{CO}_{3}^{2-}>\mathrm{Cl}^{-}\right)$, are known to be lyotropic and make the surfactant molecules more hydrophobic, and the others, on the right hand side of the series $\left(\mathrm{Br}^{-}>\right.$ $\mathrm{NO}_{3}^{-}>\mathrm{I}^{-}>\mathrm{ClO}_{4}^{-}>\mathrm{SCN}^{-}$), are known to be hydrotropic and make the surfactant molecules more hydrophilic [27-29].

We have further investigated the role of hydrophilic-hydrophobic balance in the PPO core and PEO corona interface by other additives. For this purpose, the effect of two different anions, $\mathrm{Cl}^{-}$(lyotropic) and $\mathrm{NO}_{3}^{-}$(hydrotropic) and cyclohexane as a hydrophobic additive have been tested in our reaction conditions. The addition of $\mathrm{KCl}$ or cyclohexane to the CTAB-P85 system, even at $2.5 \mathrm{CTAB} / \mathrm{P} 85$ produces a wormlike morphology, Fig. 6a and b. Note that both $\mathrm{Cl}^{-}$and cyclohexane make the P85 micelles more hydro- phobic. The $\mathrm{Cl}^{-}$ions occupy the hydrophilic regions of P85 complex micelles and enhance the contribution of the PPO block of P85, similarly cyclohexane molecules go to the PPO region of the micelles and enhance the hydrophobic interactions. However, in the presence of $\mathrm{NaNO}_{3}$, one gets a spherical morphology, Fig. 6c. The FTIR and Raman spectra of the three samples prepared using CTAB-P65 system and presence of chloride and nitrate salts are displayed in Fig. $5 c$ and d. The amount of dissolved CTAB molecules (difference spectra between the samples with and without salts display spectrum similar to dissolved CTAB) in the mesostructured silica is enhanced in the presence of both $\mathrm{Cl}^{-}$and $\mathrm{NO}_{3}^{-}$anions, Fig. 5c and d. However, the amount of dissolved CTAB is noticeably higher in the nitrate case.

The lyotropic anions occupy the aqua media close to the EO tail; however, the hydrotropic anions occupy the ethylene oxide-propylene oxide interface making the media more hydrophilic, see Scheme 1. Sachs et al. [32] have studied the dynamics of anion penetration into the hydrophobic domains of lipids where the hydrotropic anions penetrate further, thereby disrupting the hydrocarbon packing. Sachs et al.'s finding is consistent with our observations and model. The addition of salts of the lyotropic anions have an effect similar to increasing the PO units of the pluronics, enhancing the hydrophobicity of the media or the hydrophobic interactions, see Scheme 1 . Similarly, the addition of salts of hydrotropic anions makes the pluronics more hydrophilic and has the effect of increasing the EO units. The distribution of the anions in the EO units from the PO core towards EO corona influences the conformation on the EO units in the pluronics, as a result the effectiveness of the ethylene oxide groups. The distribution of the anions also affects the hydrating (hydrotropic ions wet) and dehydrating (lyotropic ions dry) of the PO units and, as a result, the conformation in the PO core and EO corona of the micelles. 

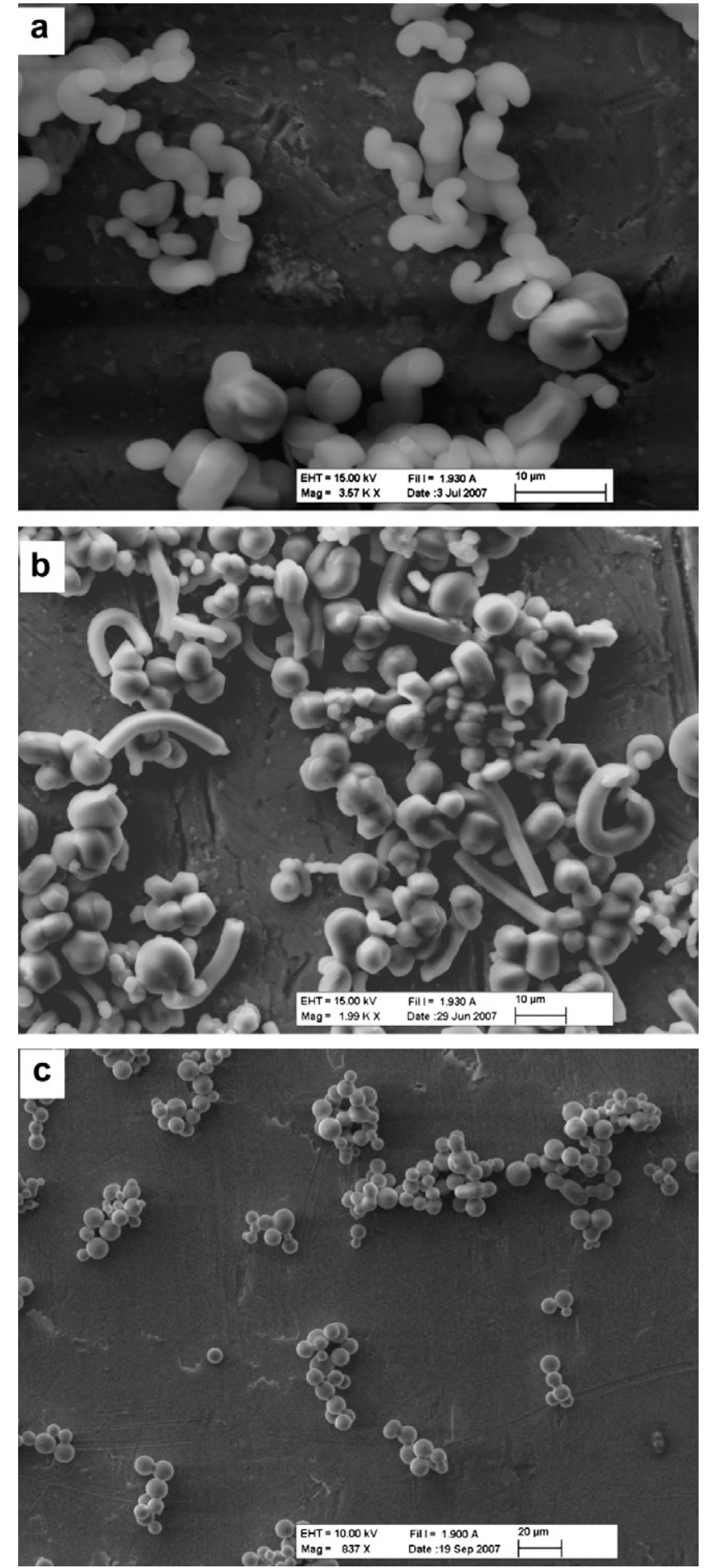

Fig. 6. The SEM images of mesostructured silica obtained from CTAB-P85 system with a CTAB/P85 mole ratio of 2.50 in the presence of (a) $1 \mathrm{~g} \mathrm{KCl}$, (b) $5 \mathrm{ml} \mathrm{Cyclo-}$ hexane, and (c) $1 \mathrm{~g} \mathrm{NaNO}_{3}$.

\section{Conclusion}

Three different morphologies were investigated under a similar reaction condition in a CTA-Pluronic-Silica system, namely spheres, wormlike particles and single crystals. Increasing the ethylene oxide units in the pluronics changes the morphology from wormlike particles to spherical particles and to single crystals in the presence of cationic surfactant. The mesostructures in the spheres and wormlike particles are 2D hexagonal while the single crystals are cubic. The addition of a cationic surfactant influences the hydrophilic-hydrophobic character of the pluronics and affects the morphology. The cationic surfactant-pluronic interaction makes the pluronics more hydrophilic and provides positive charge to their micelles, which influences the assembly of the silica species. The amount of the cationic surfactant required for one type of morphology is low in the smaller pluronics, at a CTAB/P65 mole ratio as low as 0.1 in the CTAB-P65 system, and large in the larger pluronics, at a CTAB/F127 mole ratio as high as 5.0, in the CTABF127 system. Most likely some cationic surfactant molecules remain in the solution and modify the surface of the growing silica particles.

The addition of lyotropic anions, such as $\mathrm{Cl}^{-}$and non-polar organic molecules makes the pluronic micelles more hydrophobic such that the effect is equivalent to decreasing the ethylene oxide units in the pluronics. One can obtain all three morphologies by influencing the hydrophilic character of the pluronics using salts or organic compounds in the reaction media. Further studies are required to expand the concept using other pluronics, oligo(ethylene oxide) type nonionic surfactants and may be anionic surfactant instead of cationic surfactant.

\section{Acknowledgments}

ÖD gratefully acknowledges the Scientific and Technical Research Council of Turkey (TÜBITAK) in the framework of the projects 105T224 and 105M096 and Turkish Academy of Science for the financial supports.

\section{References}

[1] T. Yanagisawa, T. Shimizu, K. Kuroda, C. Kato, Bull. Chem. Soc. Jpn. 63 (1990) 988.

[2] C.T. Kresge, M.E. Leonowicz, W.J. Roth, J.C. Vartuli, J.S. Beck, Nature 359 (1992) 710.

[3] K. Yano, Y. Fukushima, J. Mater. Chem. 13 (2003) 2577.

[4] Y. Yamada, T. Nakamura, M. Ishi, K. Yano, Langmuir 22 (2006) 2444

[5] M. Mizutani, Y. Yamada, K. Yano, Chem. Commun. 11 (2007) 1172.

[6] L. Qi, J. Ma, H. Cheng, Z. Zhao, Chem. Mater. 10 (1998) 1623.

[7] R.I. Nooney, D. Thirunavukkarasu, Y. Chen, R. Josephs, A.E. Ostafin, Chem. Mater 14 (2002) 4721.

[8] M. Mesa, L. Sierra, B. Lopez, A. Ramirez, J.L. Guth, Solid State Sci. 5 (2003) 1303.

[9] J.P. Hanrahan, A. Donovan, M.A. Morris, J.D. Holmes, J. Mater. Chem. 17 (2007) 3881.

[10] W.J.J. Stevens, K. Lebeau, M. Mertens, G.V. Tendeloo, P. Cool, E.F. Vansant, J. Phys. Chem. B 110 (2006) 9183.

[11] H. Yang, N. Coombs, G.A. Ozin, Nature 386 (1997) 692.

[12] H. Yang, G.A. Ozin, C.T. Kresge, Adv. Mater. 10 (1998) 883.

[13] B. Wang, W. Shan, Y. Zhang, J. Xia, W. Yang, Z. Gao, Y. Tang, Adv. Mater. 17 (2005) 578

[14] B. Wang, C. Chi, W. Shan, Y. Zhang, N. Ren, W. Yang, Y. Tang, Angew. Chem. Int. Ed. 45 (2006) 2088.

[15] G. Zhou, Y. Chen, J. Yang, S. Yang, J. Mater. Chem. 17 (2007) 2839.

[16] J. Wang, J. Zhang, B.Y. Asoo, G.D. Stucky, J. Am. Chem. Soc. 125 (2003) 13966.

[17] C. Yu, B. Tian, J. Fan, G.D. Stucky, D. Zhao, J. Am. Chem. Soc. 124 (2002) 4556.

[18] A.F. Demirörs, M. Arslan, Ö. Dag, Micropor. Mesopor. Mater. 98 (2007) 249.

[19] A.F. Demirörs, B.E. Eser, Ö. Dag, Langmuir 21 (2005) 4156.

[20] C. Albayrak, G. Gülten, Ö. Dag, Langmuir 23 (2007) 855.

[21] C. Guo, J. Wang, H.Z. Liu, J.Y. Chen, Langmuir 15 (1999) 2703.

[22] Y. Li, R. Xu, S. Couderc, D.M. Bloor, J.F. Holzwarth, E. Wyn-Jones, Langmuir 17 (2001) 5742.

[23] J. Jansson, K. Schillen, G. Olofsson, R.C. da Silva, W. Loh, J. Phys. Chem. B 108 (2004) 82.

[24] J. Jansson, K. Schillen, M. Nilsson, O. Söderman, G. Fritz, A. Bergmann, O. Glatter, J. Phys. Chem. B 109 (2005) 7073.

[25] D. Löf, A. Niemiec, K. Schillen, W. Loh, G. Olofsson, J. Phys. Chem. B 111 (2007) 5911.

[26] K. Aramaki, Md.K. Hossain, C. Rodriguez, M.H. Uddin, H. Kunieda, Macromolecules 36 (2003) 9443.

[27] H. Schott, J. Colloid Interface Sci. 192 (1997) 458.

[28] T. Iwanaga, M. Suzuki, H. Kunieda, Langmuir 14 (1998) 5775.

[29] C. Rodriguez, H. Kunieda, Langmuir 16 (2000) 8263.

[30] E. Leontidis, Curr. Opin. Colloid Interface Sci. 7 (2002) 81.

[31] F. Hofmeister, Arch. Exp. Pathol. Parmoakol. 24 (1888) 247.

[32] J.N. Sachs, T.B. Woolf, J. Am. Chem. Soc. 125 (2003) 8742 